\title{
GIS-based integrated evaluation of environmentally sensitive areas (ESAs) for land use planning in Langkawi, Malaysia
}

\begin{abstract}
Extensive economic growth, tourism activities and over-exploitation of resources have become the common causes of environmental degradation in Langkawi. The sudden development leap resulting from UNESCO's recognition of Langkawi Archipelago as a Global Geopark in 2007, leads to continuous conflicts between enhancing environmental protection and meeting tourism and development needs. Environmental sensitivity evaluation is a basis upon which the concept of environmentally sensitive areas (ESAs) can be practised in order to protect the environment, regulate development activities and promote sustainable land use planning. This study embarks on evaluating and classifying environmental sensitivity as well as comparing different ESA approaches applicable for land use planning in Langkawi. A GIS-based integrated evaluation model was performed on two assessment sets (Set A and Set B) using a standard grading system and weights determined with analytic hierarchy process (AHP) method. Of these sets, the former consists of selected indicators from the Malaysian integrated ESA instrument while the latter are derived from previous ecoenvironmental studies conducted in China. The projected final ESA maps of Langkawi indicate spatial distribution of four environmental sensitivity classes. More highly and moderately sensitive areas are observed in Set A, accounting for $339.15 \mathrm{~km} 2$ or $72.24 \%$ of the total land area compared to Set B with only $259.04 \mathrm{~km} 2$ or $55.18 \%$ respectively. The results also reveal large proportion of low sensitivity areas in Set A, covering areas of 117.42 $\mathrm{km} 2(25.01 \%)$. In contrast, more areas with non-sensitivity are widely distributed in Set B, occupying areas of $123.02 \mathrm{~km} 2(26.20 \%)$. Taking into consideration the natural and cultural characteristics of the islands, it is suggested that Set A is a better approach to portray current environmental concerns and to coordinate future land use planning as well as fits Langkawi's aspiration in becoming a sustainable, world-class Global Geopark. This study provides beneficial information and opportunity for reasonable rearrangement of zoning and development guidelines and strategies within sensitivity areas. It promotes effective utilization of the natural resources, minimizes negative tourism impacts and adequately highlights ecosystem functions to prosper local socio-economic growth. It also represents an early step for the design of universal ESA instruments to regulate local development activities and promote sustainable land use planning in vulnerable areas at global levels.
\end{abstract}

Keyword: Context-dependent indicators; Tropical tourism islands; Conservation strategies; Sustainable land use management; World-class Global Geopark 